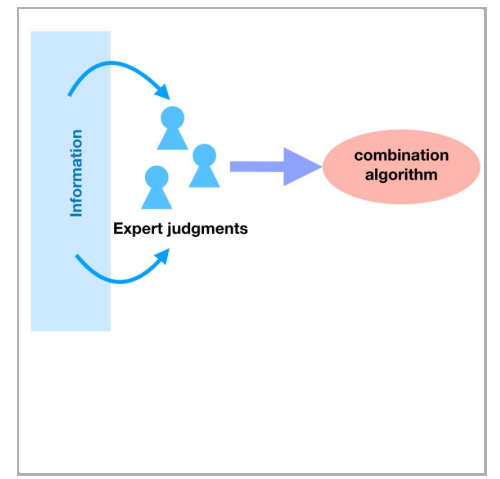

OCT 02, 2019

\title{
(3) Aggregating expert-elicited data for prediction: A scoping review of statistical methods, experiments, and applications
}

Thomas McAndrew ${ }^{1}$, Nutcha Wattanachit ${ }^{1}$, G. Casey Gibson ${ }^{1}$, Nicholas G. Reich ${ }^{1}$

${ }^{1}$ University of Massachusetts at Amherst

Tom Mcandrew

\section{DOI:}

dx.doi.org/10.17504/protocol s.io. 7 v6hn9e

\section{External link:}

http://www thomasmcandrew .com/pdf/papers/scopingRevie w_protocol_v0_3.pdf

\section{Protocol Citation: Thomas} McAndrew, Nutcha

Wattanachit, G. Casey Gibson, Nicholas G. Reich 2019.

Aggregating expert-elicited data for prediction: A scoping review of statistical methods, experiments, and applications. protocols.io

https://dx.doi.org/10.17504/p rotocols.io.7v6hn9e

License: This is an open access protocol distributed under the terms of the Creative Commons Attribution License, which permits unrestricted use, distribution, and reproduction in any medium, provided the original author and source are credited

Protocol status: Working This protocol will be used to collect articles related to methods that aggregate expert predictions.

Created: Oct 02, 2019 
Last Modified: Oct 02, 2019

PROTOCOL integer ID: 28318

Keywords: Combination forecasting, judgmental forecasting
ABSTRACT

Combining forecasts from experts, compared to a statistical model, is often more accurate when data is sparse or prone to revision. Mathematical approaches for combining forecasts span several disciplines. Economics, marketing, population dynamics, and politics all rely on expert intuition to make predictions about the future. Because expert-elicited combination forecasting is spread across different domains, researchers use a diverse set of definitions to describe the same concepts. The diversity of applications also means methodological approaches are developed in parallel rather than building on one another. Our scoping review aims to unify these varied definitions and methodological approaches, map key terminology, and summarize applications of combination forecasting, experimental designs, and methods to mathematically aggregate expert-elicited data.

We will collect articles related to methods for aggregating expert-elicited forecasts from the Web of Science database. An article is defined as in-scope if it describes eliciting expert judgments and combining them to make a prediction about a natural phenomena or future event. Articles will be considered in-scope if two committee members read the article and agree it meets the above criteria.

From the final set of in-scope articles, we will extract: the application, experimental design, metrics used to compare forecasts, and the mathematical model used to combine forecasts. Common terminology will be consolidated and discussed. We will also ask of each article a pre-specified set of questions related to combination forecasting.

This protocol prespecifies all criteria and steps for conducting a scoping review on methods for aggregating expert-elicited predictions. All aspects of this protocol were reviewed and agreed on by the coauthors, and any significant deviations from this protocol will be recorded and an amended protocol submitted. This scoping review--selecting articles in a rigorous, prespecified, and unbiased way---aims to map, classify, and summarize all aspects of literature that aggregates expert-elicited predictions.

\section{ATTACHMENTS}

scopingReview_protocol_v 0_3.pdf 